\title{
Remarks on the proof of a generalized Hartogs Lemma
}

\author{
by Evgeni Chirka (Moscow) and \\ JeAn-Pierre Rosay (Madison, Wisc.)
}

\begin{abstract}
This paper is an outgrowth of a paper by the first author on a generalized Hartogs Lemma. We complete the discussion of the nonlinear $\bar{\partial}$ problem $\partial f / \partial \bar{z}=$ $\psi(z, f(z))$. We also simplify the proofs by a different choice of Banach spaces of functions.
\end{abstract}

1. Introduction. The goal of this note is the proof of the following:

Proposition. Let $\mathcal{F}$ be the space of continuously differentiable functions defined on $\mathbb{C}^{2}$, with compact support. Then for every $\psi \in \mathcal{F}$ there exists a unique function $f$ defined on $\mathbb{C}$, tending to 0 at infinity, which is a solution to

$$
\frac{\partial f}{\partial \bar{z}}=\psi(z, f(z))
$$

This solution depends continuously on $\psi \in \mathcal{F}$ if the support of $\psi$ is restricted to be in a given compact set, and if we use the sup norm for $f$ and the $\mathcal{C}^{1}$ norm for $\psi$.

The solution of this nonlinear $\bar{\partial}$ problem is a crucial ingredient in [1]. However, this note presents some clarification and simplification. There are two differences with respect to [1]: In [1], the Proposition does not appear in this generality, and here a different choice of Banach function spaces eliminates any hard analysis in the proof (e.g. absolutely no singular integral operators theory is needed).

In order to better motivate this note, we briefly remind the reader of how the above result has been used in [1]. In [1], inspired by [2], the first author established the following theorem:

Theorem [1]. Let $f$ be a smooth function defined on $\mathbb{C}$. Assume that $f \equiv 0$ for $|z| \geq 1$. Let $h$ be a holomorphic function defined on an open set containing the union of the graph of $f$ in $\mathbb{C}^{2}\left(\left\{(z, f(z)) \in \mathbb{C}^{2}\right\}\right)$ and of the

1991 Mathematics Subject Classification: Primary 32F20; Secondary 32A10, 32D15.

Key words and phrases: nonlinear $\bar{\partial}$ problem, Hartogs Lemma. 
open set $V=\left\{(z, w) \in \mathbb{C}^{2}:|z|>1,|w|<1\right\}$. Then the restriction of $h$ to $V$ extends holomorphically to an open set containing $\left\{(z, w) \in \mathbb{C}^{2}:|w|<1\right\}$.

An immediate consequence, by means of Laurent decomposition, is the theorem which is explicitly stated in [1]:

TheOREm [1]. Let $U$ be the open unit disk in $\mathbb{C}$. Let $f$ be a continuous function on $\bar{U}$ with values in $U$, and let $S$ be its graph. Then every holomorphic function defined on a connected neighborhood of $(\partial U \times U) \cup S$ in $\mathbb{C} \times U$ extends holomorphically to the polydisk $U^{2}$.

The theorems do not extend to higher dimensions as shown in [3]. The proof of the first theorem consists in an application of the Kontinuitätssatz. Roughly speaking, one introduces a family of functions $f_{t}, 0 \leq t \leq 1$, defined on $\mathbb{C}$, depending continuously on $t$, such that: $f_{1}=f$ and $f_{0}=0, f_{t}$ tends to 0 at infinity, and $f_{t}$ is holomorphic in the neighborhood of any point $\left(z, f_{t}(z)\right)$ not in the open set where the function $u$ to be extended is defined. Such a family of functions can be defined by imposing the condition

$$
\frac{\partial f_{t}}{\partial \bar{z}}=t \varphi\left(z, f_{t}(z)\right), \quad \text { and } f_{t}(z) \text { tends to } 0 \text { as }|z| \rightarrow \infty .
$$

The function $\varphi$ is determined on the graph of $f$ by the fact that $f_{1}$ has to satisfy the equation, and $\varphi$ should have a "small" support containing this graph. The solution of the above problem (which can also be presented in terms of deformation of almost complex structures and pseudo-holomorphic curves) is given by the Proposition.

In fact, the functions $f_{t}$ should be thought of as taking values in some hull of holomorphy, on which $\varphi$ is to be defined, but the $\bar{\partial}$ problem to be solved does reduce to $\mathbb{C}^{2}$.

\section{Proof of the Proposition}

2.1. Uniqueness. Assume that $f$ and $g$ tend to 0 at infinity and satisfy $\partial f / \partial \bar{z}=\psi(z, f(z))$ and $\partial g / \partial \bar{z}=\psi(z, g(z))$. We have to show that $f=g$.

Set $h=f-g$. We immediately see that $h$ tends to 0 at infinity, $\partial h / \partial \bar{z}$ has compact support, and for some constant $C>0$,

$$
\left|\frac{\partial h}{\partial \bar{z}}\right| \leq C|h| \text {. }
$$

This implies $h=0$ for the following reason: Let $\lambda$ be defined by

$$
\lambda(z)=-\frac{\partial h / \partial \bar{z}}{h(z)}
$$

at the points $z$ where $h(z) \neq 0$, and (say) $\lambda(z)=0$ if $h(z)=0$. Set $u=\frac{1}{\pi z} * \lambda$. Then $u$ is a continuous function, tending to 0 at infinity, with $\partial u / \partial \bar{z}=\lambda$. The function $e^{u} h$ is then holomorphic on the Riemann sphere, off its zero set 
(we have simply used the technique of integrating factor). By the maximum principle, $h \equiv 0$.

2.2. Existence. We look for a solution to

$$
\frac{\partial f}{\partial \bar{z}}=\psi(z, f(z))
$$

There is a somewhat arbitrary choice of a Banach space of functions to be made for $f$. We will consider $f \in \mathcal{E}$, where $\mathcal{E}$ is the space of continuous functions $f$ on $\mathbb{C}$, with continuous $\bar{\partial}$ derivative in the sense of distributions, such that $f$ and $\partial f / \partial \bar{z}$ both tend to 0 at infinity. The space $\mathcal{E}$ is equipped with the norm $\|f\|=\sup (|f|+|\partial f / \partial \bar{z}|)$. For $f \in \mathcal{E}$ the equation $(*)$ is equivalent to

$$
f=\frac{1}{\pi z} * \psi(z, f(z))
$$

If $\alpha$ is, say, a continuous function with compact support in $\mathbb{C}$, the $\mathcal{E}$ norm of $\frac{1}{\pi z} * \alpha$ is

$$
\left\|\frac{1}{\pi z} * \alpha\right\|=\sup \left(\left|\frac{1}{\pi z} * \alpha\right|+|\alpha|\right) .
$$

We restrict $\psi$ as well, somewhat arbitrarily, by requiring $\psi \in \mathcal{F}_{0}$, where $\mathcal{F}_{0}$ is the space of continuously differentiable functions on $\mathbb{C}^{2}$ with support in the unit polydisk (no loss of generality), always equipped with the $\mathcal{C}^{1}$ norm denoted by $\|\cdot\|_{1}$.

Claim. For every $M>0$, there exists $\varepsilon>0$ such that if $\psi \in \mathcal{F}_{0}$ with $\|\psi\|_{1} \leq M$ and if $(* *)$ is solvable (with $f$ in $\mathcal{E}$ ), then for every $\psi^{\prime} \in \mathcal{F}_{0}$ satisfying $\left\|\psi-\psi^{\prime}\right\|_{1} \leq \varepsilon$ the equation $f^{\prime}=\frac{1}{\pi z} * \psi^{\prime}\left(z, f^{\prime}(z)\right)$ is solvable, and $f^{\prime}$ depends continuously on $\psi^{\prime}$.

The proposition then follows immediately. (In finitely many steps, connect 0 , for which the equation is trivially solvable, to $\psi$ ).

The claim follows from any precise version of the implicit function theorem, provided we are able to show that the map

$$
\left.\Phi: f \rightarrow f-\frac{1}{\pi z} * \psi(z, f(z))\right)
$$

satisfies the following:

(a) $\Phi$ is a continuously differentiable map from the Banach space $\mathcal{E}$ into itself.

(b) At each point $f \in \mathcal{E}, D \Phi$, the differential of $\Phi$, is an invertible linear map.

(c) $D \Phi$ depends continuously on $(\psi, f)$ in the operator norm.

(d) Finally, related to the uniformity question: If $\|\psi\|_{1} \leq M$ and $f \in \mathcal{E}$, then the operator norms of $D \Phi$ and $D \Phi^{-1}$ at $f$ are bounded by a constant 
depending only on $M$, and for every $\delta>0$, there exists $\eta>0$ depending only on $M$ such that if $\left\|\psi-\psi^{\prime}\right\|_{1} \leq \eta$ and $\left\|f-f^{\prime}\right\| \leq \eta$, then the operator norm of $D \Phi(f)-D \Phi^{\prime}\left(f^{\prime}\right)$ is $\leq \delta$.

Let us first discuss points (a)-(c). It is easy to see that $\Phi$ maps continuously $\mathcal{E}$ into itself. The $\bar{\partial}$ derivative of the function $f-\frac{1}{\pi z} * \psi(z, f(z))$ is $\partial f / \partial \bar{z}-\psi(z, f(z))$. Evaluating $\Phi$ at $f+\theta$ (think of $\theta$ as an infinitesimal increment) leads to the differential

$$
D \Phi(f, \theta)=\theta-\frac{1}{\pi z} *\left(\psi_{w}(z, f(z)) \theta(z)+\psi_{\bar{w}}(z, f(z)) \overline{\theta(z)}\right) .
$$

The operator

$$
\theta \rightarrow \frac{1}{\pi z} *\left(\psi_{w}(z, f(z)) \theta(z)+\psi_{\bar{w}}(z, f(z)) \overline{\theta(z)}\right)
$$

is a compact operator from $\mathcal{E}$ into itself. This easily follows from the fact that any bounded set in $\mathcal{E}$ clearly consists of equicontinuous functions. So $D \Phi$ is a Fredholm map of index 0 , since it is the perturbation of the identity by a compact operator. In order to check (b), it is enough to check that $D \Phi$ is injective, i.e., to check that

$$
\theta-\frac{1}{\pi z} *\left(\psi_{w}(z, f(z)) \theta(z)+\psi_{\bar{w}}(z, f(z)) \overline{\theta(z)}\right)=0
$$

implies $\theta=0$, which has been done in 2.1, since clearly $\theta$ satisfies a differential inequality $|\partial \theta / \partial \bar{z}| \leq C|\theta|$. So uniqueness is absolutely crucial in the proof of existence! (c) does not present difficulties.

Finally, we have to check (d), which is just a more precise version of (b), given by the following lemma (which itself is just a precise version of 2.1):

LEMma. For every $M>0$, there exists $C>0$ such that for every $\theta \in \mathcal{E}$, and every continuous function $\chi$ with support in the unit disk $\{z \in \mathbb{C}$ : $|z| \leq 1\}$ satisfying (pointwise) $|\chi| \leq M|\theta|$, we have

$$
\left\|\theta-\frac{1}{\pi z} * \chi\right\| \geq C\|\theta\| .
$$

Pr o of. By definition of the norm, we have to show an inequality

$$
|\theta|+\left|\theta_{\bar{z}}\right| \leq \frac{1}{C}\left(\sup \left|\theta-\frac{1}{\pi z} * \chi\right|+\sup \left|\theta_{\bar{z}}-\chi\right|\right) \text {. }
$$

Let $E$ be the subset of $\mathbb{C}$ on which $\left|\theta_{\bar{z}}\right| \geq(M+1)|\theta|$. On $E$, one even gets a pointwise inequality:

$$
\begin{aligned}
\left|\theta_{\bar{z}}-\chi\right| & \geq\left|\left(\frac{1}{M+1}+\frac{M}{M+1}\right) \theta_{\bar{z}}\right|-|\chi| \geq\left|\frac{\theta_{\bar{z}}}{M+1}\right|+|M \theta|-|\chi| \\
& \geq\left|\frac{\theta_{\bar{z}}}{M+1}\right| \geq|\theta| .
\end{aligned}
$$


Off the set $E$, this is no longer a pointwise inequality. We use the technique of integrating factor. The function $e^{u} \theta$ is holomorphic on $E^{c}$ if $u$ is obtained by solving $\partial u / \partial \bar{z}=-\theta_{\bar{z}} / \theta$ on $E^{\mathrm{c}}, u$ vanishing at infinity. Due to the support condition on $\theta_{\bar{z}},|u|$ is bounded by $K M$ (for some universal constant $K)$. By the maximum principle, the supremum of $\left|e^{u} \theta\right|$ on the set $E^{\mathrm{c}}$ is reached on the boundary of $E$ (necessarily nonempty). On this boundary $|\theta|$ is dominated by $\left\|\theta-\frac{1}{\pi z} * \chi\right\|$, as seen just above. So $|\theta|$, and therefore $\left|\theta_{\bar{z}}\right|$ (since we are on $E^{\mathrm{c}}$ ), are bounded as desired.

2.3. Discussion of uniqueness. We have pointed out in 2.2 that uniqueness as shown in 2.1 is crucial in the proof of existence. The failure of uniqueness is the exact point where the proof breaks down for higher dimensions (for which a counterexample to the theorems is known [3]). The failure of the uniqueness result, which would be needed in $\mathbb{C}^{3}$, is extremely easy to see:

With notations hopefully clear: It is not true that

$$
\left|\frac{\partial h_{1}}{\partial \bar{z}}\right| \leq C\left(\left|h_{1}\right|+\left|h_{2}\right|\right) \text { and }\left|\frac{\partial h_{2}}{\partial \bar{z}}\right| \leq C\left(\left|h_{1}\right|+\left|h_{2}\right|\right),
$$

and vanishing at infinity, imply $h_{1}=h_{2}=0$.

Indeed, take $h_{1}=h_{2}=1 / z$ for $|z|$ large, and simply arrange that $h_{1}$ and $h_{2}$ never vanish simultaneously.

\section{References}

[1] E. M. Chirka, Generalized Hartogs Lemma and the nonlinear $\bar{\partial}$-equation, in: "Complex Analysis in Contemporary Mathematics", dedicated to B. Shabat, E. Chirka (ed.), Fasis, Moscow, to appear (in Russian).

[2] S. Ivashkovich and V. Shevchishin, Pseudo-holomorphic curves and envelopes of holomorphy of two-spheres in $\mathbb{C P}^{2}$, preprint.

[3] J.-P. Rosay, A counterexample related to Hartogs phenomenon (a question by E. Chirka), preprint.

Steklov Mathematical Institute

8 Gubkin st.

GSP-1, Moscow 117966

Russia

E-mail: chirka@genesis.mi.ras.ru
Deptartment of Mathematics University of Wisconsin Madison, Wisconsin 53706 U.S.A.

E-mail: jrosay@math.wisc.edu 